\title{
Filigrane
}

Écoutes psychothérapiques

\section{Quelques réflexions sur la crise de la psychologie clinique et la formation des psychologues cliniciens}

\section{René Kaës}

Volume 17, numéro 1, printemps 2008

L’avenir du clinicien I

URI : https://id.erudit.org/iderudit/018785ar

DOI : https://doi.org/10.7202/018785ar

Aller au sommaire du numéro

Éditeur(s)

Revue Santé mentale au Québec

ISSN

1192-1412 (imprimé)

1911-4656 (numérique)

Découvrir la revue

Citer cet article

Kaës, R. (2008). Quelques réflexions sur la crise de la psychologie clinique et la formation des psychologues cliniciens. Filigrane, 17(1), 6-14.

https://doi.org/10.7202/018785ar
Résumé de l'article

La situation critique dans laquelle se trouve aujourd'hui la psychologie et la psychopathologie cliniques inspire à l'auteur quelques réflexions à partir de son expérience d'ancien professeur de cette discipline. Il expose quatre idées : $1^{\circ}$ ) la psychologie clinique n'est pas seulement menacée du dehors : elle est en crise dans sa consistance théorique, méthodologique et pratique. $2^{\circ}$ ) La psychologie clinique, notamment dans sa référence à la psychanalyse, suscite chez ceux qui la dénigrent, la haine de la réalité psychique, de l'inconscient et de ses effets de subjectivité. $3^{\circ}$ ) Il est urgent de constituer et d'enseigner une épistémologie historico-critique de notre discipline. $4^{\circ}$ ) L'extension des champs pratiques de la psychologie clinique exige quelques mesures concrètes : anticiper la demande sociale et contribuer à la formuler ; assurer la formation des psychologues cliniciens dans une association synergétique entre les universités et les institutions professionnelles. 


\title{
Quelques réflexions sur la crise de la psychologie clinique et la formation des psychologues cliniciens
}

\author{
rené kaës
}

La situation critique dans laquelle se trouve aujourd'hui la psychologie et la psychopathologie cliniques inspire à l'auteur quelques réflexions à partir de son expérience d'ancien professeur de cette discipline. Il expose quatre idées : $1^{\circ}$ ) la psychologie clinique n'est pas seulement menacée du dehors : elle est en crise dans sa consistance théorique, méthodologique et pratique. $2^{\circ}$ ) La psychologie clinique, notamment dans sa référence à la psychanalyse, suscite chez ceux qui la dénigrent, la haine de la réalité psychique, de l'inconscient et de ses effets de subjectivité. $3^{\circ}$ ) Il est urgent de constituer et d'enseigner une épistémologie historico-critique de notre discipline. $4^{\circ}$ ) L'extension des champs pratiques de la psychologie clinique exige quelques mesures concrètes : anticiper la demande sociale et contribuer à la formuler; assurer la formation des psychologues cliniciens dans une association synergétique entre les universités et les institutions professionnelles.

M a contribution à la réflexion à laquelle nous convie Filigrane est celle d'un professeur de psychologie et psychopathologie cliniques qui a enseigné au niveau universitaire pendant plus de quarante ans, dans plusieurs universités, en France et à l'étranger. J'ai aussi pratiqué la psychanalyse et contribué à sa clinique et à sa théorisation par des recherches connues.

J'ai quitté mes fonctions universitaires officielles il y a un peu plus de dix ans, mais je maintiens des liens d'enseignement et de recherche avec plusieurs universités. Toutefois, je n'ai pas connu directement les grands bouleversements qui ont accompagné, au cours de cette dernière décennie, les réformes successives et encore inachevées des études universitaires. J'ai eu la chance de rester en contact avec plusieurs de mes anciens collègues et je reçois assez régulièrement des étudiants et d'anciens étudiants qui me parlent de leurs études et de leur travail, de leur difficulté à en trouver, de leur inquiétude devant l'avenir. Nombreux sont ceux qui redoutent une disparition des psychologues cliniciens dans les structures où ils étaient traditionnellement employés, au profit de psychologues comportementalistes, cognitivistes ou de neuropsychologues. Nous avons souvent des conversations du type: «Mais comment avez-vous pensé notre formation de clinicien sans anticiper ces changements?»

Je voudrais, dans ce contexte, proposer quelques réflexions qui pointent des questions que le recul des années m'a inspirées. Elles ne s'ordonnent pas en un projet d'ensemble : ce projet reste à construire, il est aujourd'hui nécessaire. 


\section{Comment «sauver la clinique»?}

En France, récemment, un texte intitulé «Sauvons la clinique. Manifeste pour les pratiques et les formations cliniques» a alerté les diverses communautés de psychologues, de psychiatres, de psychothérapeutes et de psychanalystes à propos des menaces qui pèsent sur la psychologie clinique et sur la formation des psychologues cliniciens ${ }^{1}$. Le texte disait en substance que la formation des psychologues cliniciens est aujourd'hui mise en péril par les instances d'habilitation de leur filière, après que la formation des psychiatres a, elle aussi, été amputée de son ancrage dans la psychanalyse et dans la psychopathologie, au profit de modélisations neurobiologiques et comportementalistes. Cette attaque, précise le manifeste, exprime «la volonté de domination des partisans de l'éviction de la psychanalyse et de la psychopathologie, à tous les niveaux des organisations qualifiantes de l'enseignement et de la recherche». Les auteurs relèvent que la présence de la psychanalyse dans les institutions de soin est l'objet d'une entreprise de démolition et d'une «chasse réglée [faite] à la clinique de la subjectivité ». Le texte énumère ensuite la longue série des mesures opératoires et réductrices qui se substituent à cette clinique de la subjectivité. Jugeant vain et stérile de maintenir le refus de cette situation dans sa seule expression critique ou dans la seule dénonciation des ennemis ou dans les lamentations nostalgiques, les auteurs du manifeste dénoncent la dimension politique de cette liquidation de la clinique : il se met en place «de nouveaux dispositifs de gouvernance dans lesquelles la psychanalyse ne sera que résiduelle ou nébuleuse». Un appel à la formation d'un collectif de réflexion et d'action est alors lancé.

Assurément il faut défendre la clinique. Souscrire à ce texte de combat est une manière de lancer, une nouvelle fois, l'alerte. Mais pour défendre la psychologie clinique, il faut en repenser les fondements théoriques et pratiques, les méthodes, les champs d'application, les processus de sa transmission dans la formation des psychologues, notamment dans les universités, mais pas seulement dans les universités. Cette mise en travail concerne également les organismes professionnels qui assurent les formations complémentaires et notamment les organismes de formation de psychothérapeutes. Il nous faut aussi admettre que l'attaque ne vient pas seulement du dehors - assurément, elle est bien réelle —, et que la seule volonté de démolition ne suffit pas à mettre la psychologie clinique en péril, si elle n'était déjà en crise depuis quelques décennies. Au risque de choquer, je pense que cette crise s'est accentuée depuis la domination quelquefois abusive de la psychanalyse, ou d'une certaine manière de faire de la psychanalyse la référence exclusive de la psychologie clinique, sinon de la psychologie dans son ensemble. Non sans contradictions d'ailleurs.

Écrivant ce que je viens de dire, je voudrais dès maintenant lever l'imputation d'une ambiguïté quant à ma position. Je ne remets pas en cause la référence à la psychanalyse, je l'affirme au contraire; mais la psychologie clinique ne peut se fonder que sur des rapports de tension critique, indépassable en une synthèse, avec les références dont elle se nourrit: notamment la clinique psychanalytique, la 
clinique psychiatrique, la clinique des examens psychologiques (de Rorschach à Piaget, pour faire bref).

Je ne pense pas que l'expression critique soit vaine, non plus que se lamenter sur le monde d'hier - celui qui fut celui de ma génération - , soit en effet une bonne façon d'inventer l'avenir. Mais ce qui manque singulièrement à notre pensée, soumise à l'urgence qui caractérise les nouvelles formes de subjectivité aussi bien chez les cliniciens que chez leurs patients, c'est précisément un regard historique critique sur ce qui noue une situation de crise dans le passé avec les conjonctures actuelles qui la déclenchent. Si nous évitons ce travail, l'urgence que suscite la menace risque de masquer la crise et le travail de pensée qu'elle appelle.

Je centrerai mes réflexions sur quatre idées: la première est que la psychologie clinique n'est pas seulement menacée du dehors: elle en en crise dans sa consistance théorique, méthodologique et pratique. La seconde idée est que la psychologie clinique, notamment dans sa référence à la psychanalyse, suscite chez ceux qui la dénigrent, la haine de la réalité psychique, de l'inconscient et de ses effets de subjectivité. La troisième proposition est que nous devons, de manière pressante, constituer et enseigner une épistémologie historico-critique de notre discipline. La quatrième proposition réunit quelques mesures concrètes que rend nécessaire l'extension des champs pratiques de la psychologie clinique: il nous faut anticiper la demande sociale et contribuer à la formuler; la formation des psychologues cliniciens devrait se faire dans une association synergétique entre les universités et les institutions professionnelles.

\section{La psychologie clinique n'est pas seulement menacée, elle est en crise structurelle}

La psychologie clinique est en crise structurelle, de naissance, pourrait-on dire. Elle s'est construite entre plusieurs références, allant souvent de l'une à l'autre, sans toujours penser suffisamment la tension que générait cette pluralité de références. La référence des sciences naturelles, et plus spécifiquement la médecine, qui la fascine et qu'elle dénonce, à la fois pour sa capacité de singulariser la maladie et d'en objectiver les symptômes, et pour son écoute longuement acquise de la subjectivité et de la signification, si possible du sens du symptôme pour le malade. Cette référence conflictuelle s'inscrit dans les rémanences du dualisme corps/âme, ou corps/psyché. Le psychologue clinicien se voudrait, sans toutefois pouvoir l'être, comme le médecin au chevet du malade, mais attentif aux discours et à leur feuilletage de sens plutôt qu'aux signes et aux symptômes manifestes. Le psychologue clinicien n'a du médecin ni la formation, ni le statut social, ni le pouvoir. Il a eu beau vouloir jadis s'en donner l'apparence en revêtant la blouse blanche dans certains services, comme il le fit naguère dans les laboratoires de psychologie expérimentale des Facultés des lettres et sciences humaines, l'histoire des relations médecins - psychologues est le plus souvent celle d'une ambivalence mutuelle et de conflits dans les aires de partage du pouvoir sur le patient. Lorsque des psychanalystes, de référence lacanienne, ont dénoncé le discours 
médical comme antagoniste à celui de la psychanalyse, les psychologues cliniciens des années 1970 ont trouvé une accréditation de leur méfiance vis-à-vis des médecins: elle pouvait être fondée, et c'est un discours de psychanalystes qui l'assurait. Les psychologues cliniciens n'ont pas pour autant trouvé ce qui pouvait fonder leur identité, leur objet, leur méthode et le cadre théorico-pratique complexe qui les soutient, et qui eût été en mesure de les expliciter et les exposer à l'entendement des autres: psychologues, médecins, psychiatres, psychanalystes.

La seconde référence cruciale est précisément celle de la psychanalyse. Elle s'est mise en place sous l'effet de plusieurs facteurs. Parce que la psychanalyse a été et demeure, dans sa conception de la réalité psychique, du conflit structurant ou pathogène qui la structure, par sa prise en considération de la singularité du sujet individuel, des processus inconscients qui le lient à des ensembles intersubjectifs, un puissant modèle explicatif du fonctionnement, des troubles et des ressources de la psyché. Parce que la psychanalyse est un dispositif majeur de formation à l'écoute de soi-même et de l'autre: de l'Autre, de cet autre-là et de plus d'un autre. Jusqu'à l'époque où la référence à la psychanalyse s'est mise en place de manière prédominante dans l'enseignement des universités, à la fin des années soixante et surtout au début des années soixante-dix, la psychologie expérimentale s'imposait aux autres sous-disciplines de la psychologie comme le modèle de la psychologie scientifique, à l'exclusion de tout autre. Elle cherchait à lui en imposer le modèle de «scientificité ». Le laboratoire était la norme, et les instances universitaires de la recherche devaient prendre à leur tour ce modèle, au moins cette dénomination. J'avais essayé «élaboratoire», sans succès. J'ai connu, jeune enseignant, cette époque de domination et de méfiance vis-à-vis de la clinique et de la psychanalyse, puis l'inversion des pouvoirs, et le triomphe, aux accents de revanche, de notre conception et de notre pratique sur celles qui nous avaient opprimés. Les oppositions frontales continuaient.

Avec leurs dérives. Je n'ai pas oublié que certains courants zélotes alors triomphants de l'école de Lacan se sont retournés contre la psychologie clinique. Je me souviens aujourd'hui encore avec déplaisir et tristesse de ce milieu des années soixante-dix où la psychologie clinique était fustigée comme une clinique «clinicante », au motif que, psychologie, elle était une résistance majeure à la psychanalyse. Ce fut aussi l'époque de violentes attaques — à retardement — contre la position de D. Lagache, initiateur de l'enseignement de la psychologie clinique «dynamique » à l'université, sur l'unité de la psychologie, au prétexte que cette conception soutenait l'imaginaire de l'Un, et qu'il fallait dénoncer l'illusion que les psychanalystes (à l'université) avaient quoi que ce soit à voir et à penser avec les expérimentalistes, les généticiens, les psychosociologues.

Cette seconde référence a été, elle aussi, source de malentendus, plus graves encore, parce que non pensés, voire déniés par les enseignants, et par conséquent source de clivages et de hiatus insurmontables dans la formation des étudiants.

Sur ce point, je ne suis pas sûr d'avoir toujours réussi à tenir la position pour laquelle j'avais opté: soutenir la tension et la discontinuité entre les références 
fondatrices de la psychologie et de la psychopathologie cliniques. J'ai enseigné et formé les étudiants à la psychologie et à la psychopathologie cliniques dans la référence à la psychanalyse, et je précise : aux divers courants de la psychanalyse. Chaque fois que je l'ai pu, j'ai établi les liens avec ce que la psychologie, dans ses différentes sous-disciplines et approches, apporte comme connaissance de la psyché, comme questions et comme réponses auxquelles la psychanalyse n'a pas accès. J'ai transmis, dans ma discipline, le patrimoine vivant de la psychologie sociale lorsque j'enseignais l'approche psychanalytique des groupes.

Au cours de ma pratique, j'ai acquis une longue expérience dans le travail de supervision et d'accompagnement des équipes soignantes, dans diverses structures du soin et du traitement psychiatrique. Je peux donc témoigner, avec l'ensemble de mes collègues cliniciens, de la qualité du travail que les psychologues cliniciens ont apportée dans leur pratique référée à ce que la psychanalyse leur apprend, à ce qu'elle leur permet d'éprouver et de comprendre. J'ai vécu aussi la catastrophe qu'est, dans la conception du traitement, dans la gestion des équipes et dans le contact avec les malades, l'éradication de la formation clinique des psychiatres de la référence à un cadre de pensée psychanalytique qui avait fait ses preuves et qui avait généré d'excellentes réalisations dans le domaine des soins et de la santé mentale.

Mais je soutiens fermement que les psychologues cliniciens, ceux que les universitaires ont à former, ne sont pas à considérer a priori comme devant devenir de futurs psychanalystes. Dans plusieurs universités et non les moindres, l'enseignement de la psychologie et de la psychopathologie cliniques au cours des dernières décennies, s'est souvent limité à celui de la psychanalyse, conçue et présentée comme le modèle exclusif de la formation des psychologues cliniciens. Il est déjà regrettable que, au sein de cette ligne officielle, les débats interanalytiques n'aient pas ou guère trouvé leur place. Cette exclusivité n'est pas seulement un effet de la difficile présence, féconde et problématique, de la psychanalyse à l'université. C'est une question dès l'origine et ce n'est pas tout à fait le sujet qui nous occupe, quoique... La question est celle d'un enseignement où le risque de la «pensée unique » a pu mettre les étudiants dans une position telle que sur le terrain ils vous demandent comment, comme psychologue clinicien, «tenir une position analytique», et viennent vous voir pour s'assurer s'ils sont vraiment «condamnés » à devenir psychanalystes pour être psychologues.

Je pense que la menace qui nous frappe aujourd'hui a ses déterminations propres, actuelles, différentes de celles des années soixante ou quatre-vingt. Mais je pense aussi que cette menace a trouvé certains de ses germes et de ses rationalisations dans cette position exclusive, souvent excluante, de la psychanalyse à l'Université.

\section{La haine de la réalité psychique, de l'inconscient et de ses effets de subjectivité}

Retour du balancier? Trop simple assurément. La pensée unique qui nous est opposée comme arme idéologique est réductrice, elle ignore la complexité de la 
vie psychique d'un sujet singulier; elle est au service d'une vision du monde opératoire, simplifiée, apparemment efficace. Il faut enseigner la complexité aux psychologues cliniciens, l'intrication des composantes intrapsychiques et notamment inconscientes, avec les composantes neuropsychologiques, intersubjectives, sociales de la psyché. Le sujet existe, pluriel, dans toutes ces dimensions. Il faut leur donner des outils pour comprendre vraiment ce que disent les neurosciences et les «sciences » du comportement, et ne pas les prévenir contre elles avant de les avoir comprises, pour les discuter et, le cas échéant les réfuter.

Je suis persuadé que l'offensive politique et institutionnelle contre la psychologie clinique et la psychopathologie fondée sur la psychanalyse est une des manifestations périodiques de la haine de la réalité psychique, de la détestation de l'inconscient et de ses effets de subjectivité. Il faut assurément se défendre contre les effets dévastateurs de cette haine. Mais il ne suffit pas de se défendre, il faut proposer et mettre en œuvre de nouvelles manières de constituer et de transmettre la psychologie clinique dans toutes ses figures, dans toutes ses composantes, dans toutes ses applications, dans tous ses garants, et parmi celles-ci maintenir la référence princeps au corpus théorique et pratique de la psychanalyse.

\section{L'impérieuse nécessité de l'épistémologie historico-critique}

Une des tâches les plus importantes d'une réforme de la formation initiale des psychologues cliniciens, et sans doute la mesure vaut-elle pour toute la psychologie, est de faire une place significative à l'épistémologie de la discipline. Comment les objets de savoirs se constituent, avec quelle méthode et pour quelle pratique, dans quels contextes de connaissance et de débats avec d'autres disciplines, dans quelle situation historique, et comment il en est rendu compte dans les théories, tels sont les axes principaux d'une épistémologie critique historique qui, à ma connaissance, n'a jamais été vraiment constituée, en dépit de quelques essais courageux ici ou là.

Un principe de base de toute épistémologie est de reconnaître et de maintenir la discontinuité des champs propres à chaque discipline : la psychanalyse n'est pas la psychologie, ni la psychiatrie. C'est en creusant les spécificités des savoirs que nous pouvons tenter de les articuler, dans la tension que ne résout aucune synthèse, mais qui maintient les questionnements et la pluralité des interprétations.

Un autre principe est celui de la contextualisation des savoirs et des connaissances. Nous avons plutôt l'habitude de présenter aux étudiants des savoirs constitués, rarement le processus de leur constitution, exceptionnellement une critique interne de nos assertions. Voyez ce qu'il en résulte: la méconnaissance des origines et de l'histoire critique de sa propre discipline, l'incertitude sur ce qui la fonde, l'incapacité d'en connaître les limites et les potentialités, l'ignorance de la généalogie des idées et des découvertes qui constituent une part de leur identité. Avec cela, comment soutenir un débat avec les disciplines qui la contestent, comment éviter les prêts à porter des pensées binaires ? C'est là une grave carence de notre enseignement, et l'argument que les «programmes» sont déjà bien 
«remplis» ne tient pas: c'est nous qui les concevons. Nous devrions nous donner comme principe qu'aucun concept, aucune technique, aucun modèle ne devrait être proposé sans qu'il soit situé dans l'histoire contextualisée de son invention et de son usage - de ses usages. Ce principe s'applique bien évidemment à la psychanalyse, le plus souvent enseignée comme un corpus doctrinal dont les bordures sont faites des polémiques internes et des exclusions disciplinaires.

Cette exigence critique est, j'en suis persuadé, une source d'invention et de recherches qui ne s'engouffreraient pas dans des impasses déjà reconnues ou dans des travaux maintes fois parcourus. Ainsi pourrait être fécondée de nouveau une recherche qui trop souvent patine de ne pas avoir suffisamment claire la conscience des champs épistémiques et des objets théorico-pratiques qu'elle a pour tâche d'explorer.

\section{L'extension des champs pratiques de la psychologie clinique}

La psychologie clinique n'est pas vouée qu'à être une pratique dans les institutions psychiatriques. Ce fut un de ses berceaux et c'est encore aujourd'hui son terrain d'élection, à juste titre. Aujourd'hui, le champ s'est élargi à l'hôpital dans son ensemble et à d'autres spécialités médicales: pédiatrie, gériatrie, oncologie, chirurgie, obstétrique, cardiologie, unités de soins palliatifs, services d'urgence, etc. Les psychologues cliniciens ne sont plus seulement « au chevet du malade », ils travaillent avec eux en groupe, ils ont su faire reconnaître leur savoirfaire auprès des soignants, ils accompagnent en interne ou en consultants externes les équipes, les services, dont ils analysent les fonctionnements et les dysfonctionnements, ils soutiennent la capacité de penser les tâches respectives des différents acteurs du soin et du traitement. L'hôpital a été le foyer d'innovations psychothérapeutiques remarquables, dans lesquelles les psychologues cliniciens formés dans la référence à la psychanalyse ont joué un rôle décisif. Ils ont initié de nouvelles manières d'entendre et d'écouter, de parler de ce qui fait résistance au soin, chez les malades et chez les soignants. Mais il existe aussi des limites et des dérives dans leurs interventions. Ils ne savent généralement pas évaluer si une demande d'analyse institutionnelle relève d'une réponse de psychosociologues, de psychologues cliniciens ou de psychanalystes formés au travail institutionnel. Dans d'autres cas, la rencontre souvent violente du projet thérapeutique avec les résistances institutionnelles et les féodalités corporatives les conduit à pratiquer une analyse sauvage, au prétexte qu'il convient toujours de dire la «vérité de l'inconscient qui surgit », sans tenir compte de la capacité des destinataires à recevoir cette vérité.

Le champ d'application de la psychologie clinique est beaucoup plus large encore : il existe déjà une psychologie clinique et une psychopathologie des souffrances professionnelles, des organisations industrielles et de service. Les psychologues cliniciens ont un chantier de travail à ouvrir dans des secteurs souvent méconnus ou méprisés par les tenants de la pratique hospitalière, qui fut longtemps seule légitime et noble. Par nécessité et par extension de la demande sociale, des 
cliniciens inaugurent de manière audacieuse des activités qui ne sont pas balisées par les critères classiques auprès des populations soumises à l'exclusion sociale, aux exils et aux immigrations de toutes origines, auprès de victimes des crises et des catastrophes sociales, politiques ou dites «naturelles».

Dans l'éducation, dans des fonctions différentes de celles des psychologues scolaires, les psychologues cliniciens formés à l'écoute et au traitement des problèmes associés aux tâches éducatives (relations parents - enseignants, enseignants - institution scolaire) ont trouvé de nouveaux objets de travail, et tout autant dans les organismes de formation continue des adultes. La liste pourrait s'allonger, le recensement s'affiner. La compléter et la méditer est autant l'affaire des universitaires que des organisations professionnelles de psychologues cliniciens.

\section{Anticiper la demande sociale et contribuer à la formuler}

Mais c'est le rôle des universités de mettre en œuvre, avec des économistes et des sociologues et en contact étroit avec les organisations professionnelles, des observatoires de l'emploi, d'explorer le marché, d'anticiper la demande sociale et de formuler des offres.

Un étudiant qui entre à l'université en sort diplômé six ans après. Entre sa formation initiale et son accès au marché du travail, il s'est écoulé le temps de changements sociaux, économiques ou technologiques importants, quelquefois celui d'une mutation dans les mentalités, dans les structures sociales et dans les formes de subjectivité, comme nous l'avons vécu dans les années soixante-dix et plus récemment avec la généralisation ultra rapide de l'Internet.

\section{L'association synergétique entre universités et institutions professionnelles}

$\mathrm{Ni}$ la conception de la formation des psychologues cliniciens, ni leur formation ne peut se faire sans une association synergétique entre les universités, les institutions professionnalisantes (par exemple, les écoles de spécialisation en Argentine, au Québec ou en Italie) et les organisations professionnelles et syndicales. Chacun a son rôle spécifique à jouer, mais leur collaboration est indispensable. Voyageant dans de nombreux pays d'Europe, et en Amérique latine depuis plusieurs années, je constate, lorsque ces synergies existent, qu'elles se traduisent par des contrats entre des instituts de formation de cliniciens (psychothérapeutes, consultants) et certaines universités. Des universités européennes sont de plus en plus souvent impliquées par contrat dans ces associations synergétiques dont les objectifs de recherche, de formation et de confrontation de modèles de formation sont d'un grand bénéfice pour toutes les parties.

\section{Conclusion. Un champ ouvert}

Dans cet article, je n'ai pas fait état des dispositifs de formation qui, aujourd'hui encore, maintiennent avec détermination et efficacité les exigences d'une formation de psychologues cliniciens ouverte sur des compétences communes à 
tous les psychologues. Mon but n'était pas, non plus, de faire des propositions sur les compétences, les contenus et les parcours à mettre en place. Je me suis intentionnellement limité à quelques réflexions critiques sur les enjeux actuels de la formation des psychologues cliniciens et sur l'avenir de la psychologie et la psychopathologie cliniques, car il me semble urgent de mettre à plat les énoncés qui les fondent dans une épistémologie et dans une pratique spécifique. Parmi ces énoncés, il serait clarifiant de soutenir qu'il existe une discontinuité entre la psychologie, la psychanalyse et la psychiatrie comme branche de la médecine. C'est à cette condition que la formation des psychologues cliniciens peut trouver son assise et son éthique: dans la tension critique, hors de la confusion.

rené kaës

32 , cours de la liberté

69003 lyon

France

kaes.rene@free.fr

\section{Note}

1. http://www.sauvons-la-clinique.org/ 